\title{
Safety of users in road evacuation: design of path choice models for emergency vehicles
}

\author{
A. Vitetta, A. Quattrone \& A. Polimeni \\ Department of Computer Science, Mathematics, \\ Electronics and Transportation, Mediterranea \\ University of Reggio Calabria, Italy
}

\begin{abstract}
Within the framework of the SICURO research project, the main objective of this paper is to define the procedures to be planned and activated in emergencies in order to allow the evacuation of weak users (disabled, old persons, etc.) from the area affected by a disaster and design the optimal path for emergency vehicles to reduce evacuation times. Specification, calibration and validation of a path choice generation model in order to simulate the behaviour of emergency vehicle drivers at an urban level during an evacuation is proposed. We specify the factors that affect path choice behaviour and the two main approaches: one to one and many to one. The first regards the minimization of generalized cost of a path that connects an origin to a destination; the second instead considers the connection of one origin to many destinations. We also report some experimental results, obtained in the context of the SICURO Project, by applying the proposed model to a real road transport network at urban scale during a simulation of an evacuation.
\end{abstract}

Keywords: evacuation, path design, emergency vehicle.

\section{Introduction}

In this paper we discuss some results obtained in the SICURO research project carried out by the LAST-Laboratory for Transport Systems Analysis of the Mediterranean University of Reggio Calabria and regarding path design for emergency vehicles.

The main objective of this paper is to define the procedures to be planned and activated in emergency cases in order to allow the evacuation of weak users (disabled, old persons, etc.) from the area affected by a disaster and design the 
path for emergency vehicles. It is worth noting that there is no general path choice model completely specified and calibrated for emergency road transport systems in the event of evacuation.

This work therefore proposes the specification, calibration and validation of a path choice behavioural model at urban scale, on the basis of observation of a sample of emergency vehicle drivers who used the road network of a small town in southern Italy during an evacuation simulation.

In the simulation and design of paths for emergency vehicles, the path to follow can be chosen by two decision-makers: the driver or an external authority. In the first case the decision derives from the driver's experience and/or from a GPS navigator. In the second case the authority informs the driver of the path to follow. In order to follow the best decision, the decision-maker has to be supported by a decision support system that receives real time information from the system, designs the paths in real time, and communicates it to the driver.

To design planning co-ordination of emergency vehicles 'what to' models are considered [6]. The 'what to' approach is proposed in order to define optimal emergency vehicle distribution, in terms of vehicle number, weak users sequence to visit and design paths to minimize travel time and maximize network reliability. 'What to' models allow interventions on the supply (number of vehicles, position of the refuge areas), respecting some constraints (number of persons to save and sites to be left in).

In particular, we specify the factors that affect path choice behaviour and the two main approaches, one to one and many to one, that are commonly called, respectively, the minimum path problem and routing problem. The first regards the minimization of generalized cost of a path that connects an origin to a destination; the second instead considers the connection of one origin to many destinations. In both cases the problem is approached in three phases:

1. paths and travel times are generated using the models found in the literature;

2. the paths effectively chosen by the emergency vehicle drivers are analyzed;

3. the model is calibrated in order to maximize the degree of cover of the chosen path with those generated, each of which is obtained optimizing a function associated to a certain criterion (minimum travel time or maximum network reliability).

A congested network is assumed: the system is in an emergency condition and all the users use the network at the same time.

The paper is structured as follows: in section 2 the literature on path choice generation models is surveyed. Section 3 treats the main features of the proposed model in terms of the one to one and many to one approaches; section 4 gives the experimental results, obtained by applying the proposed model to a real road transport network at urban scale in the specific case of evacuation; in section 5 some conclusive considerations are reported.

\section{Path choice generation models}

Path choice generation models can be classified by the number of nodes to connect and the path between each origin and destination (Figure 1). In the 
specific field of emergency vehicles, the problem of path choice can be treated with two main approaches:

1. one to one, to generate paths that connect one origin to one destination;

2. many to one for the connection of one origin to many destinations.

The many to one problem is an extension of the one to one problem because in each pair of nodes to visit a one to one problem has to be solved. Hence the one to one problem is presented first. The many to one problem is presented later and can be considered the optimization of a chain of one to one problems.

Below we specify the main features of the one to one approach, distinguishing the two main phases of generating the choice set and choosing an alternative (section 2.1) and many to one approach (section 2.2).

\begin{tabular}{|c|c|c|}
\hline \begin{tabular}{|c|}
$\begin{array}{c}\text { Nodes } \\
\text { Paths } \\
\text { Number }\end{array}$ \\
to connect
\end{tabular} & One to One & One to Many or Many to One \\
\hline 1 & Shortest Path & Routing \\
\hline more than 1 & $\begin{array}{c}\text { k- Shortest Path } \\
\alpha-\text { Shortest Path } \\
\text { not Outstanding Path }\end{array}$ & $\begin{array}{c}\mathrm{k}-\text { Routing } \\
\alpha-\text { Routing } \\
\text { not Outstanding Path Routing }\end{array}$ \\
\hline
\end{tabular}

Figure 1: Approaches for path choice generation models.

\subsection{One to one}

The one to one approach gives the probability of every path being chosen, from those perceived as admissible, between each origin to each destination. The problem of one to one path choice is complex due to the large number of existing alternative $(\mathrm{o}, \mathrm{d})$ pairs, also on small-size networks, and their overlapping.

These difficulties are treated in various studies in which complete model specification of path choice is articulated in two phases:

1. generation of the choice set, that is the possible alternatives $[1,3,13,15$, 18];

2. path choice among the alternatives belonging to the choice set generated $[1,3,5,7,8,16,18]$.

Regarding the choice set generation, there are different approaches in the literature (figure 2): in an exhaustive approach, all the loopless analytical paths on the network are available and belong to the single choice set for all users; in a selective approach, only some available paths represent attractive choice alternatives.

In the second case, the generation of available paths can be obtained following three different approaches: with the monocriterion approach, deterministic or probabilistic multi-criteria approach.

Regarding the monocriterion approach the available paths are obtained by the satisfaction of a single criterion. In order to generate the minimum path for the criterion it is necessary to minimize trip disutility measured with a single 
attribute (time, distance, etc.) or construct a covered function of the criterion. The parameters of the covered function must be calibrated, maximizing, for example, the overlapping factor between the generated and the observed paths.

Alternatively, it is possible to use the multi-criteria approach according to which the available paths are obtained by satisfying some criteria calibrated by maximizing the overlapping factor between the generated and observed paths, similar to the monocriterion approach.

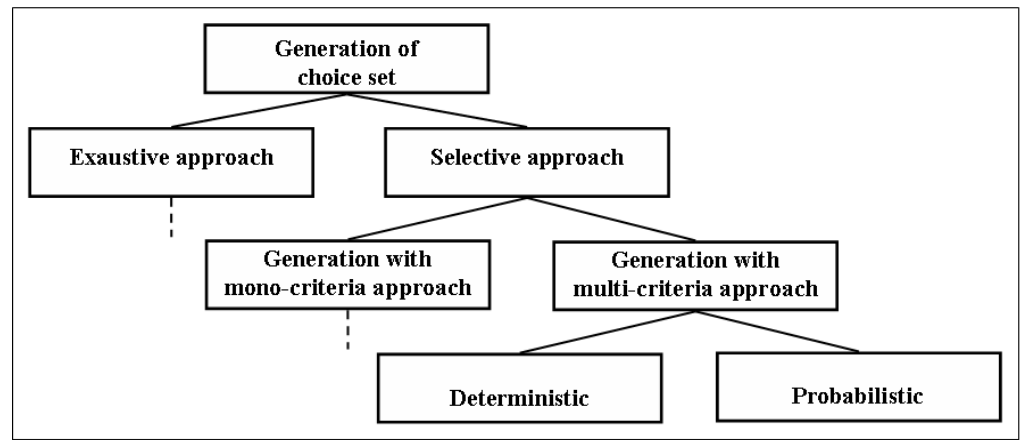

Figure 2: Approaches for choice set generation.

As regards path choice, most of the models proposed in the literature belong to the family of Random Utility Models (RUMs). Based on assumptions on the random residual of the utility perceived by users, the models can have different specifications, of which those most commonly used for path choice are Multinomial Logit, C/DC-Logit, Path Size and Probit [4, 8, 14, 15, 18, 19].

\subsection{Many to one}

Concerning the many to one approach the problem of path choice generation can be specified as a routing problem. The routing problem regards the necessity to visit a certain number of nodes (or links) in a given sequence, leaving from an origin and returning to it, with the respect of some constraints (i.e. number of users to visit and their localization, number of vehicles and their capacity, etc.) and the aim to optimize an Objective Function OF. The OF can depend on travel time, monetary cost or number of vehicles.

The on routing problems are extensive, especially in the freight transport field $[9,10,21]$.

In the case of emergency vehicles [12] the problem is treated under the assumption that a general vehicle covers a given zone, assigning to the vehicles a sequence of nodes to visit according to the characteristics of the users served.

Araz et al. [2] study an emergency system (ambulances and anti-fire vehicles) so as to diminish travel time and maximize the area covered and the number of users saved.

In the presence of a natural disaster Takahashi et al. [20] propose a model that simulates the variations in user path choice behaviour when, due to a calamitous 
event, the road network has limited accessibility. The model entails estimating the variation in costs incurred by users who are forced to modify their path choice.

Therefore, the routing problem regards the need to visit a certain number of nodes (or links) in a given sequence, leaving from an origin and returning to it, so as to optimize an objective function OF. The OF can depend on travel time, monetary cost and number of vehicles. Routing problems can be grouped into two distinct classes: node routing problems and link routing problems.

Whereas the node routing problem consists in visiting some nodes leaving from an origin, the link routing problem regards cover of some network links. A further distinction can be made within the classes defined above, in particular in the case of node routing: the vehicle routing problem (classical or asymmetric) and salesman problem.

In the case of link routing we can distinguish the Chinese postman problem (oriented or non-oriented) and rural postman problem.

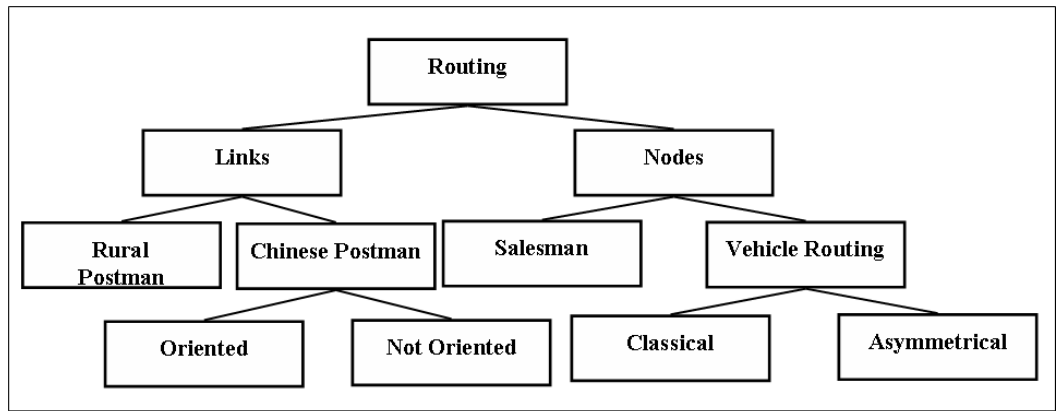

Figure 3: Approaches to the routing problem.

\section{Proposed model}

The proposed model is used to analyze the behaviour of a sample of emergency vehicle drivers in terms of path choice on a road network at urban level in the case of evacuation. The path choice generation models are simulated with two approaches: one to one and many to one. In both cases the paths generated satisfy a function specified on the basis of observation of emergency vehicle driver behaviour. Below we specify the proposed model with the one to one approach (section 3.1) and many to one approach (section 3.2).

\subsection{One to one}

Manski defines in 1977 the probability of a generic path $\mathrm{k}$ as the sum, on all the choice sets which contain the alternative, of the product between the probability of the choice set and the conditional probability of choosing path $\mathrm{k}$ given the choice set. 


$$
p^{n}(k)=\sum_{I_{i}^{n}} p^{n}\left(k, I_{i}^{n}\right)=\sum_{I_{i}^{n}} p^{n}\left(k / I_{i}^{n}\right) \cdot p^{n}\left(I_{i}^{n}\right)
$$

where

$p^{n}\left(I_{i}^{n}\right)$, probability of the choice set $I_{i}^{n}$

$p^{n}\left(k / I_{i}^{n}\right)$, choice probability of the generic path $\mathrm{k}$

Concerning the generation of the choice set, the selective approach followed with multicriteria, described in section 2.1, is used to define for every $(o, d)$ pair (refuge area - weak user residence) a choice set that consists of several paths $k$, each of which is generated in respect to a certain criterion. The criteria used depend on the factors that affect the emergency vehicle drivers' behaviour. These criteria are: minimum travel time and maximum network reliability.

For both the criteria, relative to every link $i j$ of the network, a covered function is defined. The function is dependent on travel time and unknown parameters. The general structure characterizing the covered functions for every criterion $h$ is:

$$
\begin{gathered}
h_{(i j) k}=\beta_{R(i j)} \cdot t_{R(i j) k} \text { if the link } i j \text { belongs to the reserved network } \\
h_{(i j) k}=\beta_{N R(i j)} \cdot t_{N R(i j) k} \quad \text { otherwise }
\end{gathered}
$$

where

$\beta_{R(i j)}, \beta_{N R(i j)}$ are parameters calibrated for both criteria $h$

$t_{R(i j) k}, t_{N R(i j) k}$ is travel time on link $i j$ belonging to path $k$ and respectively to the reserved or non-reserved network. Table 1 reports the parameters relative to the covered functions which have the above general structure.

Table 1: $\quad$ Parameters of the covered functions for the criteria.

\begin{tabular}{lcc}
\hline Criterion $(h)$ & $\beta_{R(j)}$ & $\beta_{N R(i j)}$ \\
\hline Minimum travel time & 1 & 1 \\
Maximum reliability & 1 & $1 / \Phi_{\mathrm{ij}}$ \\
\hline
\end{tabular}

The network reliability $\Phi_{i j}$ of link $i j$, relative to the time interval $T$, is defined as the conditional probability that vehicle speed does not decrease during $T$ to the point where the density limit is reached, given that the level at instant zero is equal to the average speed corresponding to the flow rate in stable flow conditions [11].

\subsection{Many to one}

Concerning the many to one approach the vehicle routing problem, described in section 2.2, is used to define a choice set that consists of several paths $k$, each of which optimizes an objective function and connects one origin (refuge area) to many destinations (weak user residences). 
Given the following notation:

$L=\{(i, j), \forall i, j \in N\}$, link set;

$f_{i j}$, ordinary vehicle flows;

$\delta$, amplifying factor;

$e_{i j}$, emergency vehicle flows;

$r_{i}$, demand on node $i$;

$v$, emergency vehicle;

$N V=\{1,2, \ldots, N V\}$, number of emergency vehicles;

$b$, emergency vehicle capacity;

$x_{i j v}$, variable that is equal to $l$ if the link $i j$ is used by vehicle $v$, zero otherwise;

$y_{i v}$, variable that is equal to 1 if the node $i$ is already visited by vehicle $v$, zero otherwise;

$\Phi_{i j}$, reliability on link $i j$ (see section 3.1 );

the vehicle routing problem can be expressed with the following general optimum problem.

$$
\text { Objective Function: Minimizing: } \sum_{(\mathrm{i}, \mathrm{j}) \in \mathrm{L}} h_{i j}\left(\mathrm{f}_{\mathrm{ij}}+\delta \mathrm{e}_{\mathrm{ij}}\right) \sum_{v=1}^{N V} x_{i j v}
$$

variable: $x_{i j v} \quad \forall(\mathrm{i}, \mathrm{j}) \in \mathrm{L} ; \mathrm{v}=1,2, \ldots, \mathrm{NV}$

subject to:

$$
\begin{gathered}
\sum_{\mathrm{v}=1}^{\mathrm{NV}} \mathrm{y}_{\mathrm{iv}}=1 \quad \forall \mathrm{i} \in \mathrm{Z}, \mathrm{i}=1,2, \ldots, \mathrm{n} \\
\sum_{\mathrm{v}=1}^{\mathrm{NV}} \mathrm{y}_{0 \mathrm{v}}=\mathrm{NV} \\
\sum_{\mathrm{j}} \mathrm{r}_{\mathrm{i}} \mathrm{y}_{\mathrm{iv}} \leq \mathrm{b} \quad \mathrm{v}=1,2, \ldots, \mathrm{NV} \\
\mathrm{y}_{\mathrm{iv}} \in\{0,1\} \quad \forall \mathrm{i} \in \mathrm{N} \\
x_{i j v} \in\{0,1\} \mathrm{i} \in \mathrm{N} ; \quad \forall(\mathrm{i}, \mathrm{j}) \in \mathrm{L} \\
\sum_{j \in Z} x_{i j v}=\sum_{j \in Z} x_{j i v} \quad \forall \mathrm{i} \in \mathrm{Z}, \mathrm{v}=1,2, \ldots, \mathrm{NV}
\end{gathered}
$$

where

concerning minimum travel time: $h_{i j}=t_{i j}$, travel time on link $i j$ distinguishing reserved or non-reserved networks;

concerning maximum reliability: $h_{i j}=t\left(\mathrm{f}_{\mathrm{ij}}\right) / \Phi\left(\mathrm{f}_{\mathrm{ij}}\right)$, ratio between travel time and reliability on link $i j$, both depending on link ordinary flow $f_{i j}$.

\section{Experimentation}

The proposed model, described in the previous section, was specified, calibrated and validated for an emergency system, studied with regard to path choice on a road transport network at urban scale. The model was calibrated on the basis of emergency vehicle driver monitoring during a simulation of evacuation, carried out by the Transport System Analysis Laboratory (LAST) in Melito Porto Salvo 
(Calabria, southern Italy) within the ambit of the SICURO Project. The paths chosen by the driver sample were monitored using a GPS (Global Positioning System) on board emergency vehicles and video cameras deployed on the network. The network consists of all the main roads (37 nodes and 66 links).

As regards generation of the choice set, the unknown parameters of covered and objective functions were calibrated by maximizing the degree of overlapping of the chosen paths by the sample with the generated paths.

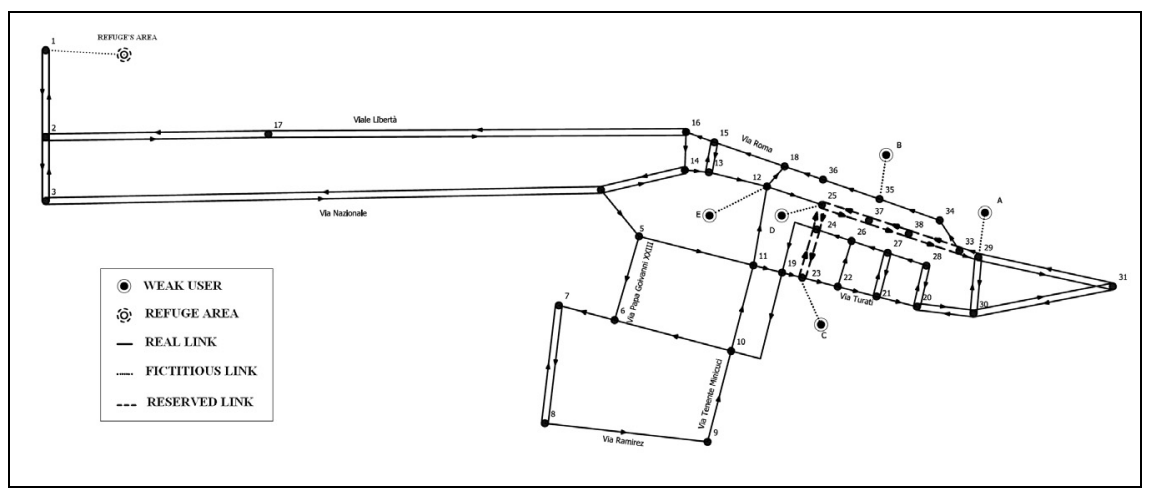

Figure 4: Melito Porto Salvo road network with localization of weak users and the refuge area.

Given one origin coinciding with the refuge area (RA) and the residence localization of five (A, B, C, D, E) weak users to rescue (Figure 4), we analyzed the observed paths. The drivers chose two routing paths with only one emergency vehicle: in the first routing two weak users were rescued in the second three. The total evacuation time observed was approximately 47 minutes. We then simulated with the proposed model the observed path, obtaining almost the same results. This demonstrates the model's goodness-of-fit. We also designed the optimal path with the proposed model, considering both the approaches previously specified, One to one and Many to one, assuming different scenarios in terms of number of emergency vehicles and their capacity. With the one to one scenario, assuming only one emergency vehicle in order to rescue one weak user at a time, we obtained a reduction in evacuation time of about $15 \%$. The same result was achieved with the many to one approach, assuming only one emergency vehicle with a greater capacity in order to rescue all five users at a time. Under another hypothesis (two emergency vehicles that move at the same time and rescue all weak users with two many to one routings) the total evacuation time is reduced approximately by $20 \%$.

\section{Conclusion}

In this paper some results of the SICURO research project are given and in particular the problem of emergency vehicle drivers' path choice at urban scale during an evacuation are treated. Path choice generation behavioural models 
were specified and calibrated for the road transport system of an Italian town based on monitoring evacuation procedures of some weak users during a simulation. These calibrations supplied some valid results and provide sound indications on the emergency vehicle driver path decisional process in the urban context and in the specific field of an evacuation when a disaster occurs.

Preliminary calibrations for the Italian urban network also show that significant improvements in terms of evacuation time saving can be obtained by designing emergency vehicle paths and defining the main characteristics of the supply system for the weak users such as the number of vehicles and their capacity.

In the future, the model should be further analyzed through the use of a more extensive network and database, and different scenarios should be proposed by also varying the refuge area number and localization.

\section{References}

[1] Antonisse, R. W., Daly, A. J., and Ben Akiva, M., Highway assignment method based on behavioural models of car driver's route choice, Transportation Research Record (1220), pp 1-11, 1985.

[2] Araz, C., Selim, S., Ozkarahan, I., A fuzzy multi-objective covering-based vehicle location model for emergency services. In Computers \& Operations Research 34, pp 705-726, 2007.

[3] Ben Akiva, M., Bergman M. J., Daly A. J., Ramaswamy, R., Modelling interurban route choice behaviour, Proceedings of the $9^{\text {th }}$ International Symposium on Transportation and Traffic Theory, VNU Science Press, pp 299-330, 1984.

[4] Ben Akiva, M., Lerman, S. R., Discrete choice analysis: theory and application to travel demand, MIT Press, Cambridge, Mass, 1985.

[5] Ben-Akiva, M. and Bierlaire, M., Discrete choice methods and their applications to short term travel decisions, Chapter for the Transportation Science Handbook, Preliminary Draft, 1999.

[6] Cantarella, G. E., Vitetta, A., The multi-criteria road network design problem in an urban area. Transportation, vol. 33, pp. 357-588, 2006.

[7] Cascetta, E., Nuzzolo, A., Biggiero, L., Analysis and modelling of commuters departure time and route choices in urban networks, Proceedings of the $2^{\text {nd }}$ International Seminar on Urban Traffic Network, Capri, Italy, 1992.

[8] Cascetta, E., Nuzzolo, A., Russo, F., Vitetta, A., A new route choice logit model overcoming IIA problems: specification and some calibration results for interurban networks, Proceedings of the $13^{\text {th }}$ International Symposium on Transportation Traffic Theory, Jean-Baptiste Lesort ed., Pergamon Press, 1996.

[9] Cho, Y.J., Wang, S.D., A threshold accepting meta-heuristic for the vehicle routing problem with backhauls and time windows. In Journal of the Eastern Asia Society for Transportation Studies, V 6, pp. 3022 - 3037, 2005 . 
[10] Chiou, Sw, Integrating the inventory management and Vehicle Routing Problems For Congested Urban Logistics Network. In Journal of the Eastern Asia Society for Transportation Studies, V 6, pp. 3038 - 3051, 2005.

[11] Ferrari, P., The traffic control on motorways. In Proceedings of seminar Traffic image processing - State of development and possibility of utilization, Agostino Nuzzolo (ed.), 1991.

[12] Yang, S., Hamedi, M., Hagani, A., Online Dispatching and Routing Model for Emergency Vehicles with Area Coverage Constraints. In Transportation Research Record: Journal of the Transportation Research Board Issue Number 1923, 2005

[13] Morikawa, T., A hybrid probabilistic choice set model with compensatory and non compensatory ruler, Proceedings of the $7^{\text {th }}$ WCTR, Sydney, Australia, 1996.

[14] Ortuzar, J. d. D. and Willumsen, L. G., Modelling transport, Wiley and Sons, England, 1990.

[15] Russo, F. and Vitetta, A., Networks and assignment models for the Italian national transportation system, Proceedings of the $7^{\text {th }}$ WCTR, Sydney, Australia, 1995.

[16] Russo, F. and Vitetta, A., An assignment model with modified Logit, which obviates enumeration and overlapping problems, Transportation 30, pp 117-201, 2003.

[17] Russo, F. and Vitetta, A., La ricerca di percorsi in una rete: Algoritmi di minimo costo ed estensioni, Franco Angeli, Milan, Italy, 2006.

[18] F. Russo, A. Vitetta, A. Quattrone, Route choice modelling for freight transport at national level. Proceedings of the European Transport Conference, Strasbourg, 2007.

[19] Sheffy, Y., Urban transportation networks, Prentice Hall, Englewood Cliff, NJ, 1985.

[20] Takahashi et al, Disaster impact analysis of limited access through traffic network model: the case of $m t$. Usu eruption. In Proceedings of the Eastern Asia Society for Transportation Studies, V. 5, pp. 2441 - 2453, 2005.

[21] Taniguchi, E., Ando, N., An experimental analysis on probabilistic vehicle routing and scheduling with its. In Journal of the Eastern Asia Society for Transportation Studies, Vol. 6, pp. 3052 - 3061, 2005. 